\title{
A CAC Algorithm for ATM Networks Supporting Different QoS Classes
}

\author{
M.Ajmone Marsan ${ }^{1}$, A.Bianco ${ }^{1}$, C.Casetti ${ }^{1}$, P.Castelli ${ }^{2}$, R.Lo Cigno ${ }^{1}$, \\ M.Mellia ${ }^{1}$, M.Munafó ${ }^{1}$
}

${ }^{1}$ Dipartimento di Elettronica, Politecnico di Torino

Corso Duca degli Abruzzi, 24 - 10129 Torino - Italy

e-mail: \{ajmone,bianco, caset ti,locigno,mellia,munafo\}@polito.it

${ }^{2}$ CSELT

Via G.Reiss Romoli, 274 - 10148 Torino - Italy

e-mail: Paolo.Castelli@cselt.it

\begin{abstract}
This paper describes a connection admission control (CAC) algorithm for ATM networks supporting different Quality of Service (QoS) classes, and illustrates its effectiveness with simulation results considering both the call-level and the cell-level dynamics.

The CAC algorithm groups connection requests in three different QoS classes: i) Class 1: with stringent CLR (Cell Loss Ratio) and CDV (Cell Delay Variation) requirements; ii) Class 2: with stringent CLR requirements, but no need for CDV guarantees; iii) Class U: with no need for guarantees on either CLR or CDV. Both Constant Bit Rate (CBR) and Variable Bit Rate (VBR) connections can request admission as either Class 1 or Class 2, depending on their QoS requirements. Unspecified Bit Rate (UBR) and Available Bit Rate (ABR) connections instead normally request admission as Class $\mathrm{U}$.

The investigation of the effectiveness of the CAC algorithm is based on the simulation of an ATM network with parking lot topology, and with variable parameter values.

The relationship between the proposed CAC algorithm and equivalent bandwidth (EB) CAC algorithms described in the literature is discussed.
\end{abstract}

\section{Keywords}

ATM, CAC, Traffic Management, Quality of Service

*This work was supported in part by a research contract between Politecnico di Torino and CSELT, in part by the Italian Research Council (CNR) and in part by the Italian Ministry for University and Research.

The original version of this chapter was revised: The copyright line was incorrect. This has been corrected. The Erratum to this chapter is available at DOI: 10.1007/978-0-387-35353-1_28 


\section{INTRODUCTION}

In competitive telecommunication markets, the success of a network operator largely depends on two factors: i) the Quality of Service (QoS) provided to the end users, and ii) the cost of the service utilization for the end users. These two factors on the one hand determine the end user satisfaction, and on the other hand are determined by the effectiveness of the algorithms adopted by the network operator for the exploitation of the network resources.

In order to leave space for the competition among network operators, standardization bodies in many cases refrain from the standardization of those algorithms whose impact on the efficient utilization of network resources is crucial. Several examples of such algorithms are possible, and some of the most important surely concern algorithms for the implementation of the Connection Admission Control (CAC) functions.

The goal of CAC functions is achieving the best possible exploitation of network resources, while guaranteeing that the QoS remains above the level promised to the end user. CAC functions thus play a central role in guaranteeing the customer satisfaction.

In the particular case of ATM networks, the role of CAC functions is even more delicate than in traditional circuit-switched networks, since the variability of the telecommunication services offered by the network implies a very wide range of data rates and several QoS requirements.

Several different proposals of CAC algorithms for ATM networks appeared in the literature; the most widely used approaches are based either on the definition of an equivalent bandwidth for each connection, or on the actual measurement of the bandwidth used by active connections $[1,2,3,4,5]$.

Although it is intrinsically impossible to define the optimality of a CAC algorithm for ATM networks, some requirements for a "good" CAC algorithm are evident. A good CAC algorithm for ATM networks should

1. achieve high resource utilization;

2. allow the exploitation of statistical multiplexing;

3. guarantee the QoS promised to the end user;

4. be robust with respect to traffic fluctuations;

5 . be based on simple traffic descriptors;

6 . be simple to implement;

7. provide immediate answers.

In this paper we illustrate and evaluate by simulation a simple $\mathrm{CAC}$ algorithm for ATM networks that explicitly considers the presence of connections with different $\mathrm{Q} O S$ requirements.

QoS requirements are confined to a set of predefined classes, and the traffic descriptor used by the $\mathrm{CAC}$ is independent from the link or node charac- 
teristics, so that there is no need to fine tune the algorithm when network characteristics change.

The proposed CAC is suitable for an adaptive implementation since its behavior depends on very few parameters, whose physical significance is easily understood and can be modified according to cell-level QoS measurements. Adaptive versions of the CAC algorithm, however, are not discussed in this paper, and are left for further study.

The paper is organized as follows. The CAC algorithm is described and discussed in Section 2. The simulation tools used for the assessment of the effectiveness of the CAC algorithm are described in Section 3, while the networking scenario that is used as a testbed for the simulation experiments is presented in Section 4. Simulation results are presented in Section 5. Finally, Section 6 ends the paper with some concluding remarks.

\section{THE CAC ALGORITHM}

The key goal of the CAC algorithm is to decide about the admission of connections with different QoS requirements, using a set of simple equations and a very limited amount of information, namely only the peak cell rate (PCR) and sustainable cell rate (SCR) of admission-requesting connections.

Specifically, three QoS classes are considered:

Class 1: with stringent CLR (Cell Loss Ratio) and CDV (Cell Delay Variation) requirements;

Class 2: with stringent CLR requirements, but no need for CDV guarantees; Class $U$ : with no guarantees on either CLR or CDV.

The QoS as defined by ITU [6] is described through a fairly complicated set of parameters, each one ranging on a large number of possible values. It is clear that the simple 3-class QoS scenario we are assuming cannot support the complete set of QoS vectors defined in [6]. It must be argued, however, that most users and applications cannot be expected to be capable of describing the QoS they need in such fine detail, and most networks will probably not offer the whole range of possible QoS alternatives.

The mapping of ATM tranfer capabilities, namely Constant Bit Rate (CBR), Variable Bit Rate (VBR), Unspecified Bit Rate (UBR), and Available Bit Rate (ABR), onto QoS classes is not trivial. However, one can argue that CBR and real-time VBR (rt-VBR) should be admitted as Class 1, non-real-time VBR (nrt-VBR) as Class 2, and best-effort services like ABR and UBR as Class U. CBR connections can, in principle, require admission as Class 2; however, a CBR connection that is not guaranteed a low CDV probably will not appeal to many users. Therefore, we may safely assume that only nrt-VBR calls request admission as Class 2. 
The CAC algorithm is run for each link that a connection should use; the rule to decide about the admission of a connection is quite simple:

- For each link, Class 1 and 2 connections are accepted if:

$\sum_{C_{1}} \mathrm{PCR}+\sum_{C_{2}} \mathrm{MfCR} \leq \alpha C$

where $C_{1}$ and $C_{2}$ are the sets of connections in Classes 1 and 2, respectively, $C$ is the link capacity, and $\alpha$ is a protection coefficient $(\alpha \leq 1)$ that can be set so as to avoid that the whole link capacity is used by connections of Classes 1 and 2. MfCR stands for Modified Cell Rate, and is a parameter characterizing connections with a cell rate value midway between PCR and SCR, according to a factor $\gamma$; that is:

$$
\mathrm{MfCR}=\mathrm{SCR}+\gamma(\mathrm{PCR}-\mathrm{SCR})
$$

Since connections can exhibit a substantially different behavior for different degrees of burstiness, picking a similar range of $\gamma$ when deciding about the admission of more or less bursty connections can result in a misleading characterization of their behavior; $\gamma$ should thus be regarded as a function of the burstiness. In our CAC scheme, we introduce a simple relationship between $\gamma$ and the connection burstiness (computed as $B=\mathrm{PCR} / \mathrm{SCR}$ ), in the form of $\gamma=\gamma_{0} / B$. "Sensible" values for $\gamma_{0}$ range between 0 and $B$ (clearly, $\gamma_{0}<0$ would result in bandwidth under-allocation, and $\gamma_{0}>$ $B$ would entail a bandwidth over-allocation for bursty connections, thus reducing link utilization). Thus we have:

$$
\mathrm{MfCR}=\mathrm{SCR}+\frac{\gamma_{0}}{B}(\mathrm{PCR}-\mathrm{SCR})
$$

A discussion of the relationship between the expression of MfCR with frequently used equivalent bandwidth expressions is included in the following subsection. Incidentally, note that the value of $\gamma_{0}$ does not directly affect the admission of CBR connections, for which PCR=SCR.

- Class U calls are accepted if on each link:

$$
\sum_{C_{U}} \mathrm{PCR} \leq \beta\left(C-\sum_{\mathcal{S}_{\mathrm{CBR}}} \mathrm{PCR}-\sum_{\mathcal{S}_{\mathrm{VBR}}} \mathrm{SCR}\right)
$$

where $C_{U}$ is the set of connections in Class $\mathrm{U}, \mathcal{S}_{\mathrm{CBR}}$ and $\mathcal{S}_{\mathrm{VBR}}$ are the sets of CBR and VBR connections, and $\beta$ is a bandwidth utilization coefficient, possibly greater than 1 , affecting the amount of leftover bandwidth that can be allocated to Class U calls. 
As can be understood from the above description, $\alpha$ and $\beta$ are CAC parameters independent from the traffic mix, whereas $\gamma_{0}$ is more closely related to the characteristics of bursty connections.

\subsection{Modified Cell Rate and Equivalent Bandwidth}

The expression of MfCR in equation (3) is based only on the PCR and SCR traffic descriptors, which are provided by the user with the request of connection setup.

In order to relate the MfCR definition with frequently used equivalent bandwidth definitions, consider the case of ON/OFF VBR source models, according to which users have fixed cell generation rate $p=$ PCR during 'ON' periods, and are silent during 'OFF' periods.

The average offered cell rate of such users is $m=\mathrm{SCR}$, that can be expressed as:

$m=p P(\mathrm{ON})$

where $P(\mathrm{ON})$ is the probability of sources being in the ON period.

Moreover, for such user models, the variance of the offered cell rate can be written as:

$\sigma^{2}=p^{2} P(\mathrm{ON})-m^{2}=p^{2} \frac{m}{p}-m^{2}=m p-m^{2}=m(p-m)$

Hence we have:

$\mathrm{SCR}=m$

$(\mathrm{PCR}-\mathrm{SCR})=p-m=\frac{\sigma^{2}}{m}$

and, recalling that $B=p / m$, from (3) we get:

$\mathrm{MfCR}=m+\frac{\gamma_{0}}{B} \frac{\sigma^{2}}{m}=m+\frac{\gamma_{0}}{p} \sigma^{2}$

The CAC defined in (1) in this case states that VBR connections are accepted if:

$\sum \mathrm{MfCR} \leq \alpha C$ 
that is, if:

$$
\sum \frac{1}{\alpha}\left(m+\frac{\gamma_{0}}{p} \sigma^{2}\right) \leq C
$$

This shows that with this type of sources the proposed CAC algorithm based on MfCR exhibits significant similarities with frequently used CAC algorithms based on equivalent bandwidth $(E B)$ expressions, such as the one proposed by Lindberger (see Chapter 5 in [4] and [5]), in which the connection is accepted if:

$\sum E B \leq C$

with:

$E B=a m+\frac{b}{C} \sigma^{2}$

where $C$ is the link capacity and $a, b$ are coefficients whose values Lindberger evaluated so as to achieve a cell loss probability smaller than a given threshold, and empirically found to be well matched to the following formulas:

$a=1-\frac{\log _{10} P_{\text {loss }}}{50}=1-\frac{L_{p}}{50}$

$b=-6 a L_{p}=\frac{6 L_{p}^{2}}{50}-6 L_{p}$

with $L_{p}=\log _{10} P_{l o s s}$, and $P_{\text {loss }}$ the maximum acceptable cell loss probability. In his analysis, Lindberger had to adopt a model including a number of simplifications, the most relevant being the assumption of a superposition of an infinite number of sources generating fixed-length and fixed-rate bursts according to a Poisson process, and the presence of a bufferless queue.

The advantage of Lindberger's expression is that it allows to explicitly account for a cell loss probability requirement, but the similarity of the CAC rules based on MfCR and $E B$ allows the selection of a value for $\gamma_{0}$ such that the same performance requirement is met.

Indeed, we can observe that, with the assignment:

$$
\begin{aligned}
\alpha & =\frac{1}{a} \\
\gamma_{0} & =\frac{b p}{a C}
\end{aligned}
$$


(11) and (12) become identical.

Simulation results will be used in later sections to verify whether this approach is actually capable of guaranteeing the expected upper bound on the cell loss probability in realistic networking environments.

\section{THE SIMULATION TOOLS}

The investigation of the effectiveness of the CAC scheme we just described is possible at both the cell level and the call level by using an integrated simulation environment for ATM networks developed at Politecnico di Torino, under contract with CSELT.

Simulations are run by two distinct simulation tools working at the two different time scales: namely, ANCLES (Atm Networks Call LEvel Simulator) and CLASS (Cell Level Atm Services Simulator). Either simulator computes metrics relevant to the time scale it is working at: while ANCLES allows the estimation of call blocking probabilities and average link loads for different CAC and routing schemes, CLASS addresses such performance parameters as cell loss probabilities or cell delay distributions, beside assessing the effectiveness of traffic management techniques such as shaping, policing and ABR algorithms.

In our integrated simulation environment, call-level dynamics are explored by ANCLES; when a "critical" configuration in terms of congestion level and duration is found, its traffic pattern is fed to CLASS, which carries on the analysis at the cell level.

However, the simulation control is never actually switched back and forth between the two simulators: the intervention of an operator, selecting the most "critical" configurations, is advisable, given the cost in terms of CPU time involved by cell-level simulations. Therefore, a set of ANCLES runs is usually followed by a number of CLASS simulations, according to the operator's choices.

A more detailed description of ANCLES/CLASS interactions can be found in [9].

\section{THE SIMULATED SCENARIO}

The setting we chose to investigate the behavior of the CAC scheme described in Section 2 is quite simple.

Fig. 1 depicts the ATM network configuration under examination. A network topology of the type usually referred to as parking lot is used. The capacity of all user-node and node-node links is set to $150 \mathrm{Mbit} / \mathrm{s}$, while the length of each link varies so as to equalize the end-to-end delays between sources and destinations.

As shown in Fig. 1, each node (numbered 1 through 4 ) is linked to 3 call 


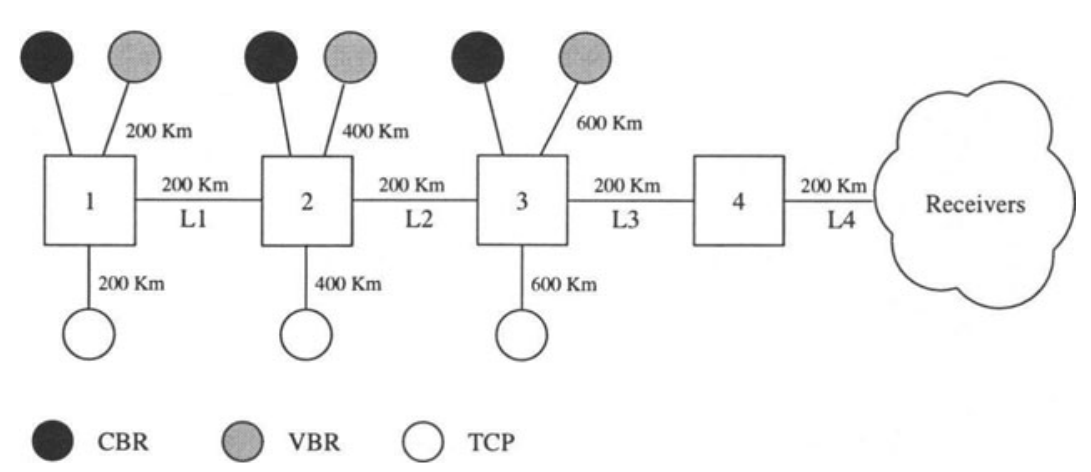

Figure 1 Simulated ATM network configuration

generators - or users (the circles in Fig. 1). Of course, the exact number of connections opened by the users connected to a node (possibly zero) varies as the call-level simulation experiment proceeds, and is determined by both the offered traffic load and the CAC algorithm; however, users behave as concentrators and the number of links connected to each node remains constant.

Three types of users are considered: CBR, non real-time ON/OFF VBR, and Best Effort. One user of each type is connected to nodes 1, 2, and 3, Best Effort users are modeled as greedy TCP traffic generators. The traffic generated by TCP users is either shaped, or controlled according to the principles of the ABR ATM transfer capability. CBR users activate connections with constant bit rate $(2 \mathrm{Mbit} / \mathrm{s})$, and with holding times determined by i.i.d. exponential random variables averaging at 1000 seconds. ON/OFF VBR users open calls with PCR equal to $10 \mathrm{Mbit} / \mathrm{s}$; once activated and admitted to the network, VBR calls hold for an exponentially distributed period, with average 1000 seconds; the SCR of VBR connections is variable: results are derived for the cases SCR $=1 \mathrm{Mbit} / \mathrm{s}$, and SCR $=100 \mathrm{kbit} / \mathrm{s}$, corresponding to the burstiness values $B=10$ and $B=100$, respectively. The execution of calllevel simulation runs requires no other information; however, when cell-level simulations are run, the characterization of the ON/OFF VBR sources is also needed: the durations of ON periods is taken to be exponentially distributed with average $\mu_{\mathrm{ON}}=50 \mathrm{~ms}$; the OFF periods duration is also exponentially distributed, with mean $\mu_{\mathrm{OFF}}=(B-1) \cdot \mu_{\mathrm{ON}}$.

For what concerns TCP users, each node is requested to support connections with $10 \mathrm{Mbit} / \mathrm{s}$ PCR and average holding time equal to 60 seconds. TCP users are assumed to activate long ftp file transfers that last for the whole connection holding time. However, the actual average transfer rate on all TCP connections is determined by the TCP protocol dynamics and by their interaction with CBR and VBR traffic, that have a higher priority.

The data transferred by each connection, regardless of the source position, 
are routed to a sink connected to node 4 , creating a bottleneck on the link between node 3 and node 4 .

The allocation of connections within QoS classes generally depends on the QoS requirements of the end user. For the sake of simplicity we assume that all real time connections are CBR, while all non real time connections are VBR. For this reason, in the simulation runs that produced the results that will be discussed below, all connections generated by CBR users are mapped onto Class 1, all VBR connections are mapped onto Class 2, and all TCP connections are accommodated in Class U. The traffic load offered by Class 1 calls is twice that offered by Class 2 calls. The nominal load of TCP connections is instead quite hard to define: TCP connections declare only their PCR, but the average traffic generated by them during cell-level simulations depends on the network load and the transfer capability supporting Class $U$ calls, as already noted.

For what concerns parameters of interest to cell-level simulations, ATM switches are assumed to have an output queued, non blocking architecture and each output interface comprises three separate output buffers for cells belonging to connections of Classes 1,2, and U. A fixed priority scheme is employed to serve buffers, Class 1 being the highest priority, Class $U$ the lowest. The buffer size for Class 1 traffic is equal to 64 cells, since high priority, real time traffic needs buffer space only for the resolution of cell scale contention; the buffer size for Class 2 and Class $U$ traffic is equal; two different values are used in simulation experiments: either 1024 or 2048 cells.

\section{NUMERICAL RESULTS}

The CAC parameters used in our simulations are $\alpha=1$, and $\beta=4$, while different values of $\gamma_{0}$ are examined.

The nominal load cumulatively offered by Class 1 and Class 2 traffic, normalized with respect to the capacity of the congested link is used as an independent parameter for the call-level simulations. As already pointed out, the load offered by TCP connections cannot be estimated a priori, however the TCP call generation rate is set so as to overload the network in almost any condition, since the use of a large value of $\beta$ accounts for a very permissive admission policy for best-effort, Class $U$ traffic.

The simulation results we collected at the call level can be divided into two sets:

- curves of the blocking probabilities for CBR, VBR and TCP connections, versus the nominal load offered to the bottleneck link by CBR and VBR connections

- curves of the utilization of the bottleneck link by CBR and VBR connections, versus the nominal load offered to the bottleneck link by CBR and VBR connections 
After having chosen the nominal loads that result in blocking probabilities approximately equal to a target value (set to 0.01 in our simulations), some critical congestion configurations are saved by the call-level simulator; simulations at the cell level then provide the following results:

- CDV, CLR and average load of the bottleneck link for CBR and VBR connections;

- throughput, goodput and CLR for TCP connections.

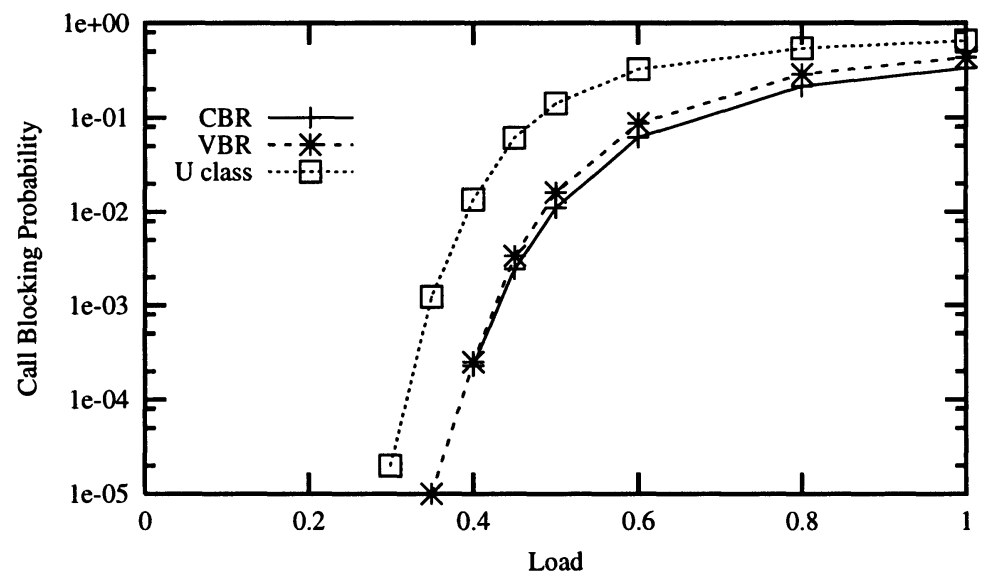

Figure 2 Blocking probabilities vs. normalized offered load; $\gamma_{0}=2, \mathrm{~B}=10$

The first set of results (Figs. 2, 3, 4 and 5) shows call blocking probabilities referring to CBR, VBR and U Class connections, as a function of the traffic load offered to the bottleneck link by CBR and VBR connections normalized with respect to the link capacity $(150 \mathrm{Mbit} / \mathrm{s})$. The four different figures are derived for variable values of the burstiness of VBR connections $(B=10$ and $B=100)$, and of the parameter $\gamma_{0}\left(\gamma_{0}=2\right.$ and $\left.\gamma_{0}=4\right)$.

With $\gamma_{0}=2$ (Figs. 2 and 3), the blocking probabilities for U Class connections are significantly larger than for CBR and VBR connections. Instead, the blocking probabilities for CBR connections are smaller than for VBR connections when the latter have burstiness $B=10$, but the opposite is true when the burstiness of VBR connections grows to $B=100$. This is due to the fact that with $B=10$ the MfCR of VBR connections is larger than the PCR of CBR connections, but it becomes smaller for $B=100$.

It can be noted that blocking probabilities for CBR and VBR connections with different burstiness appear to reflect the bandwidth allocation determined by (1), resulting in blocking probabilities for CBR almost ten times 


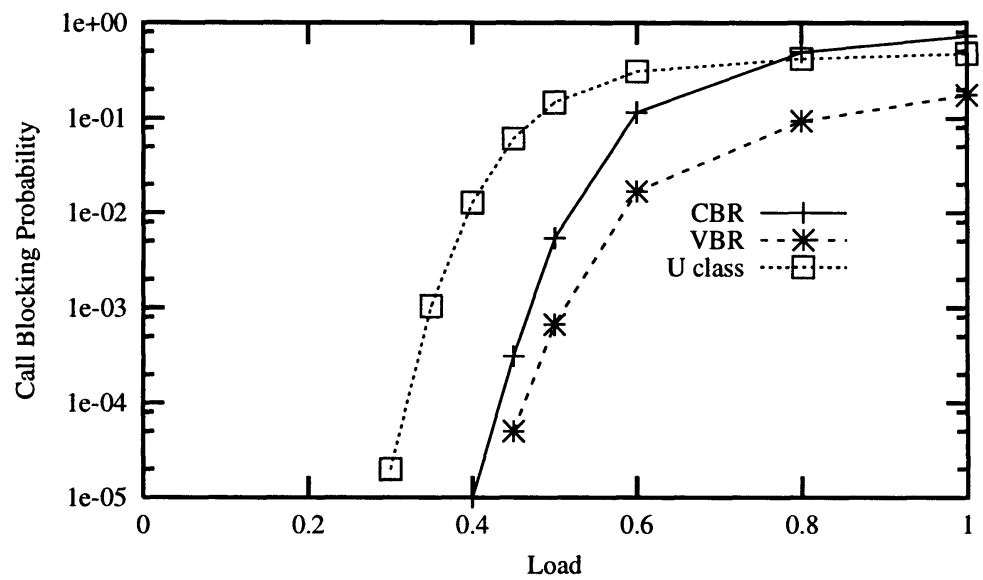

Figure 3 Blocking probabilities vs. normalized offered load; $\gamma_{0}=2, \mathrm{~B}=100$

higher than VBR when $B=100$ (this can easily be explained if we consider that $\mathrm{PCR}_{\mathrm{CBR}}=2 \mathrm{Mbit} / \mathrm{s}$ and $\left.\mathrm{MfCR}_{\mathrm{VBR}}=0.298 \mathrm{Mbit} / \mathrm{s}\right)$.

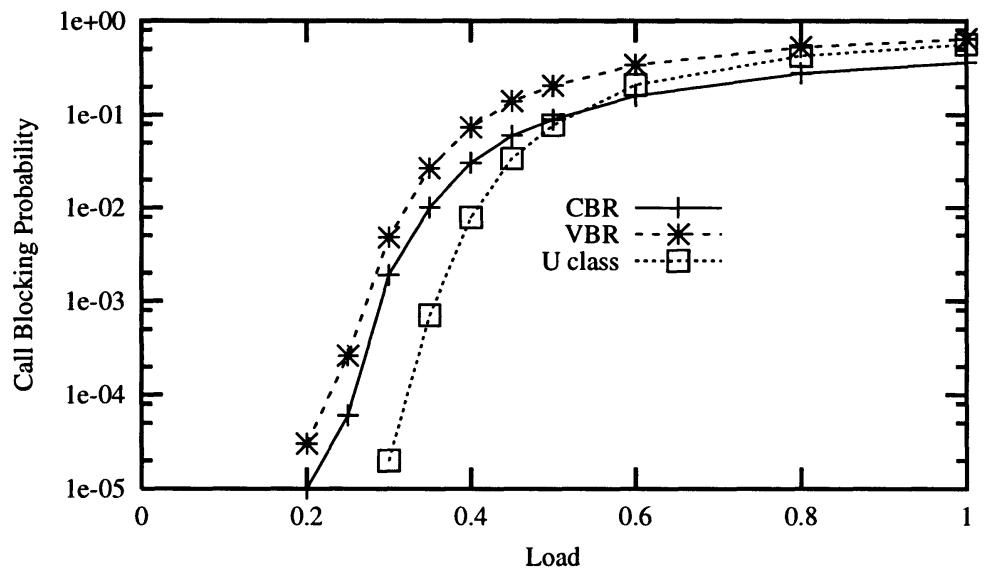

Figure 4 Blocking probabilities vs. normalized offered load; $\gamma_{0}=4, \mathrm{~B}=10$

With $\gamma_{0}=4$ (Figs. 4 and 5), the blocking probability for U Class connections is reduced to values smaller than for CBR and VBR connections. However, increasing the value of $\gamma_{0}$ entails two significant drawbacks. First of all, increasing $\gamma_{0}$ leads to increased blocking probabilities for CBR and VBR connections, as seen by the comparison of Figs. 2 and 4, or Figs. 3 and 5. Second, increasing $\gamma_{0}$ results in a lower utilization of the bottleneck link by CBR 


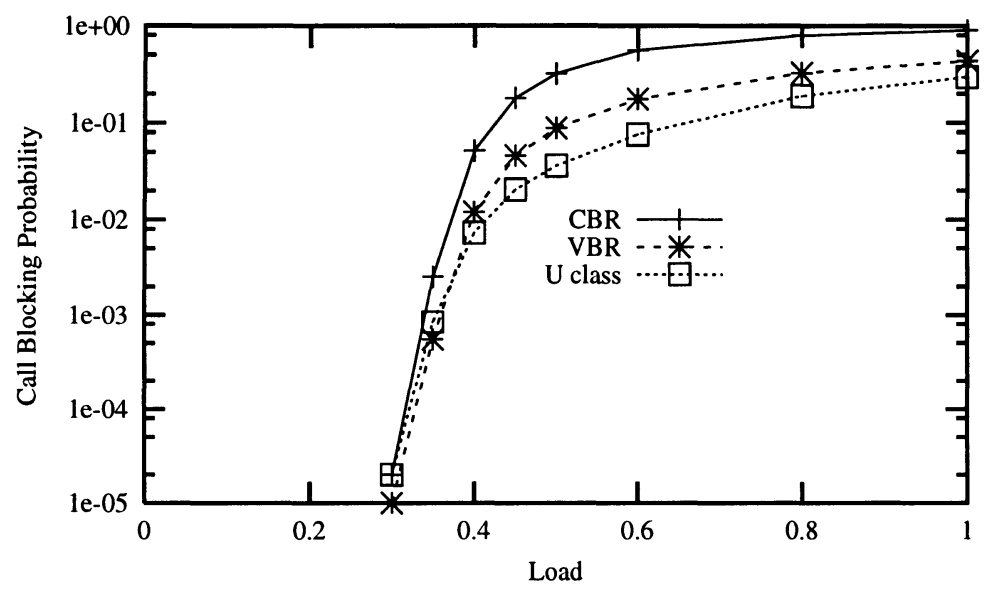

Figure 5 Blocking probabilities vs. normalized offered load; $\gamma_{0}=4, \mathrm{~B}=100$

and VBR connections (the traffic that should generate the largest percentage of revenues for the network operator). This can be seen in Figs. 6 and 7, that show curves of the bottleneck link utilization by CBR and VBR connections versus the nominal load offered to the bottleneck link by CBR and VBR connections, for values of $\gamma_{0}$ ranging from 0 to $B$, the value of the burstiness of VBR sources (recall that for VBR connections, with $\gamma_{0}=0, \mathrm{MfCR}=\mathrm{SCR}$; instead, with $\gamma_{0}=B, \mathrm{MfCR}=\mathrm{PCR}$ ). The curves show that almost half of the available capacity, and sometimes more, remains unused, unless the chosen value for $\gamma_{0}$ is quite small, close to $\gamma_{0}=0$. Note however that the proposed $\mathrm{CAC}$ algorithm can be seen to be capable of providing much better utilization of the bottleneck link than a PCR-based CAC (curves with $\gamma_{0}=B$ ).

Achieving a high utilization of the bottleneck link is just half of the task of a CAC algorithm; the second half of the task is guaranteeing the QoS of connections. In order to verify that this goal is also achieved, the simulation of the ATM network at the cell level is necessary. This was done by selecting a nominal network load such that the blocking probabilities for CBR and VBR connections are close to 0.01 , and mapping TCP connections on either a UBR service with shaping at $10 \mathrm{Mbit} / \mathrm{s}$, or an $\mathrm{ABR}$ service. In the latter case, ABR control is achieved using the ERICA (Explicit Rate Indication for Congestion Avoidance) algorithm [10] with target utilization 0.98 .

Cell-level simulations were run for 5 different configurations generated by the call-level simulations; these configurations were deemed to be the worst cases observed during several hours of simulated network activity. This analysis was restricted to the case $\gamma_{0}=2$ that is more critical than $\gamma_{0}=4$. Results obtained in the 5 different scenarios are then averaged. Cell-level results are reported in Table 1 . The eight columns with numerical values refer to ABR 


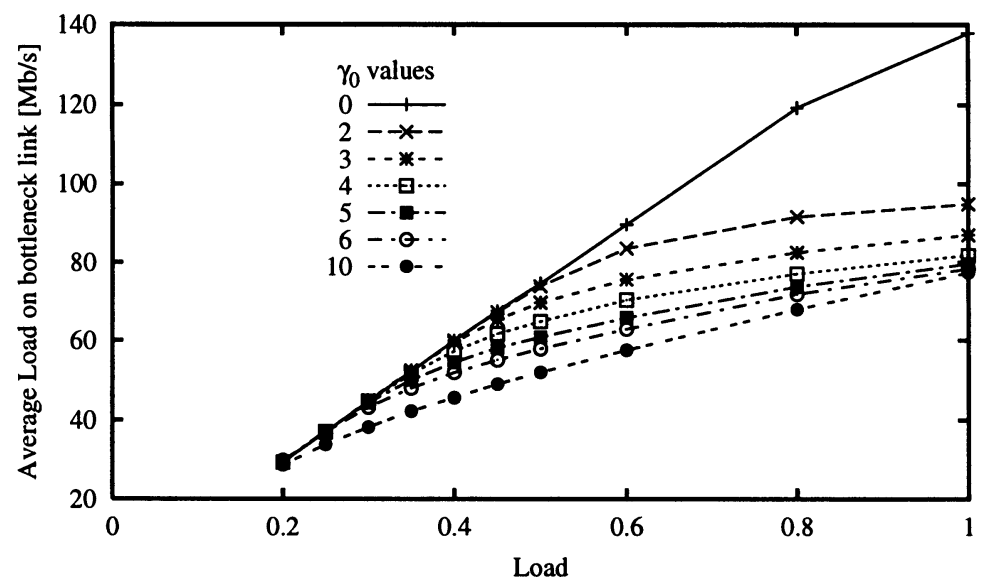

Figure 6 Average load on bottleneck link vs. normalized offered load, for several values of $\gamma_{0} ; \mathrm{B}=10$

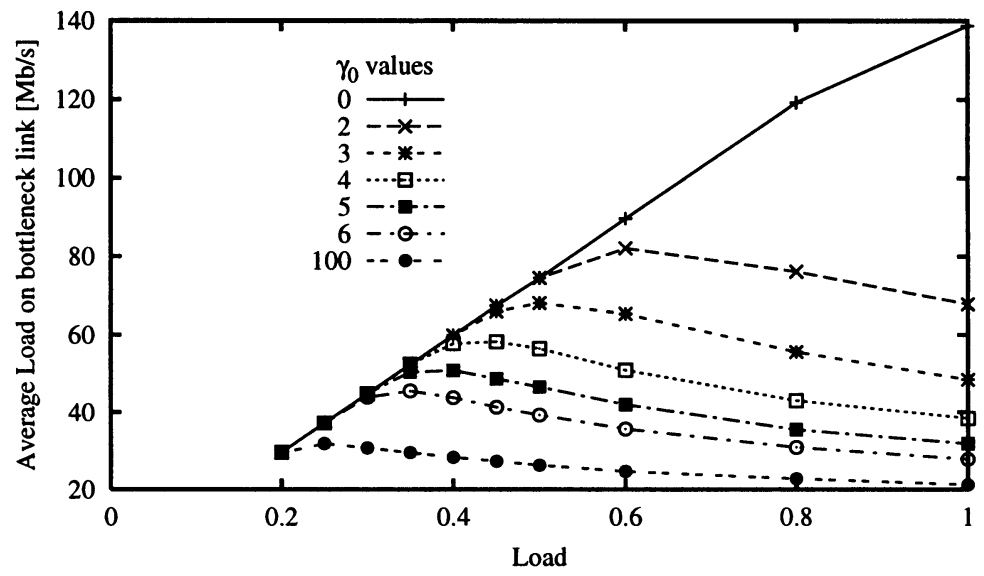

Figure 7 Average load on bottleneck link vs. normalized offered load, for several values of $\gamma_{0} ; \mathrm{B}=100$

and UBR, with either $B=10$, or $B=100$, and with Class 2 and Class U buffers whose capacity is either 1024 or 2048 cells.

The first three rows provide throughput $(S)$ results in Mbit/s for CBR and VBR connections, while for TCP connections the goodput $\left(S_{G}\right)$ is shown. Goodput is defined as the useful throughput: goodput values are obtained by discarding all corrupted and duplicated segments, that is, considering only those segments that are useful to reconstruct the end user information. The 


\begin{tabular}{|c|c|c|c|c|c|c|c|c|c|}
\hline & & \multicolumn{4}{|c|}{ Buffer 1024} & \multicolumn{4}{|c|}{ Buffer 2048} \\
\hline & & \multicolumn{2}{|c|}{ ABR } & \multicolumn{2}{|c|}{ UBR } & \multicolumn{2}{|c|}{ ABR } & \multicolumn{2}{|c|}{ UBR } \\
\hline & & $B=10$ & $B=100$ & $B=10$ & $B=100$ & $B=10$ & $B=100$ & $B=10$ & $B=100$ \\
\hline CBR & $S$ & 79.2 & 66.0 & 79.2 & 66.0 & 79.2 & 66.0 & 79.2 & 66.0 \\
\hline VBR & $S$ & 22.6 & 22.8 & 23.0 & 22.8 & 22.7 & 22.8 & 22.7 & 22.7 \\
\hline $\mathrm{TCP}$ & $S_{G}$ & 42.6 & 56.4 & 25.7 & 30.8 & 44.1 & 57.7 & 32.4 & 38.3 \\
\hline \multirow[t]{2}{*}{ TCP } & $B_{w}$ & 1.2 & 0.5 & 18.3 & 22.6 & 0.4 & 0.2 & 13.5 & 17.2 \\
\hline & $B_{u}$ & 4.4 & 4.3 & 3.8 & 7.8 & 3.6 & 3.3 & 2.2 & 5.8 \\
\hline CBR & CLR & 0 & 0 & 0 & 0 & 0 & 0 & 0 & 0 \\
\hline VBR & CLR & $1.6 \mathrm{E}-4$ & $1.7 \mathrm{E}-4$ & $1.8 \mathrm{E}-4$ & $2.0 \mathrm{E}-4$ & $2.7 \mathrm{E}-5$ & $8.9 \mathrm{E}-5$ & $2.8 \mathrm{E}-5$ & $9.6 \mathrm{E}-5$ \\
\hline $\mathrm{TCP}$ & CLR & $8.1 \mathrm{E}-3$ & $3.2 \mathrm{E}-3$ & $1.1 \mathrm{E}-1$ & $8.8 \mathrm{E}-2$ & $2.9 \mathrm{E}-3$ & $1.3 \mathrm{E}-3$ & $6.7 \mathrm{E}-2$ & $5.4 \mathrm{E}-2$ \\
\hline CBR & $\mu_{d}$ & 0.7 & 0.7 & 0.7 & 0.7 & 0.7 & 0.7 & 0.7 & 0.7 \\
\hline VBR & $\mu_{d}$ & 26.9 & 6.0 & 28.8 & 6.1 & 37.8 & 6.1 & 38.8 & 6.1 \\
\hline $\mathrm{TCP}$ & $\mu_{d}$ & 1521.4 & 799.5 & 4910.3 & 3605.3 & 2014.7 & 874.9 & 10321.3 & 7731.8 \\
\hline CBR & $\sigma_{d}$ & 1.4 & 1.4 & 1.4 & 1.4 & 1.4 & 1.4 & 1.4 & 1.4 \\
\hline VBR & $\sigma_{d}$ & 297.1 & 24.2 & 311.4 & 25.4 & 499.5 & 24.1 & 512.7 & 24.7 \\
\hline $\mathrm{TCP}$ & $\sigma_{d}$ & 3648.7 & 2012.4 & 4700.2 & 3375.2 & 5070.9 & 2488.6 & 8404.8 & 6310.3 \\
\hline
\end{tabular}

Table 1 Results obtained with CLASS for the 5 congested configurations generated by ANCLES with $\gamma_{0}=2$

throughput of CBR and VBR connections depends only marginally on the service class used for TCP connections, thanks to the use of separate buffers and fixed priorities. The goodput of TCP connections, instead, is drastically influenced by the service class choice: the ABR goodput is significantly larger than the UBR goodput.

The fourth and fifth rows provide results in Mbit/s for the amount of bandwidth wasted by TCP connections $\left(B_{w}\right)$ (the resources used by the network to deliver information useless for the TCP receiver) and for the portion of bandwidth that goes unused on the bottleneck link $\left(B_{u}\right)$. The results show that the bandwidth wasted by TCP connections is quite small in the case of ABR, but it is about $2 / 3$ of the TCP goodput in the case of UBR with smaller buffers, and about $1 / 2$ with larger buffers.

The following three rows report loss probabilities for the three types of connections. The fact that loss probabilities are zero for CBR connections, in spite of the very small available buffers, is obvious, thanks to the priority that is given to this class of traffic. Loss probabilities are also quite small for VBR connections and decrease with buffer increase; this is due to the fact that buffers are larger, and that this traffic has priority over TCP traffic. Loss 
probabilities for TCP traffic are instead quite high, specially when the UBR service class is used.

The last rows report results for the average of the variable part of the cell delay $\left(\mu_{d}\right)$ (that is, the difference between the actual cell delay and propagation and processing delays), and the standard deviation of the same quantity $\left(\sigma_{d}\right)$. All results are in $\mu \mathrm{s}$. Averages and standard deviations of delay variations are quite small for CBR connection. This is quite a positive result, that, together with the null CLR values, indicates that the QoS requirements for CBR connections are satisfied.

Consider now VBR connections; values of the average and standard deviation of delay variations are significantly larger, but this type of traffic can tolerate such performance. The fact that the values of the average and standard deviation of delay variations with $B=100$ are much smaller than with $B=10$ is due to the reduction of the CBR traffic from 79 to $66 \mathrm{Mbit} / \mathrm{s}$ and to a stable VBR traffic load; this results in shorter queues at the Class 2 buffer within the switch, hence in shorter delays. Indeed, the increase in TCP traffic that can be obseved when $B$ grows from 10 to 100 does not impact the VBR traffic performance thanks to the priority service discipline.

Coming finally to TCP connections, we see that the values of the averages of delay variations are about four times larger with UBR than with ABR. Standard deviations are instead similar with the two service classes.

In summary, the numerical results provided by cell-level simulations indicate that the QoS requirements of CBR and VBR connections can be satisfied with the proposed CAC, at least if the buffer for Class 2 traffic is large enough, and the value of $\gamma_{0}$ is chosen carefully, a task that might not be easy a priori. As far as TCP connections are concerned, a great difference is observed between the use of the UBR and the ABR service classes. With ABR, the obtained QoS is quite good, and the utilization of the network resources is very satisfactory. Instead, with the UBR service class, the obtained QoS is significantly worse, but, even more important, the exploitation of the available network resources shows dramatic inefficiencies.

\subsection{CAC and Cell Loss Ratio Guarantees}

As we observed in Section 2.1, the CAC algorithm that we considered can be reduced to the equivalent bandwidth CAC algorithm proposed by Lindberger, under the assumption of ON/OFF sources, exploiting expressions (16) and (17) for the choice of the values of $\alpha$ and $\gamma_{0}$.

Lindberger's equivalent bandwidth CAC algorithm defines its parameters so as to be able to guarantee a specified cell loss ratio under rather stringent conditions, that mainly consist in the assumptions of a superposition of an infinite number of sources generating fixed-length and fixed-rate bursts according to a Poisson process, and the presence of a bufferless queue. 

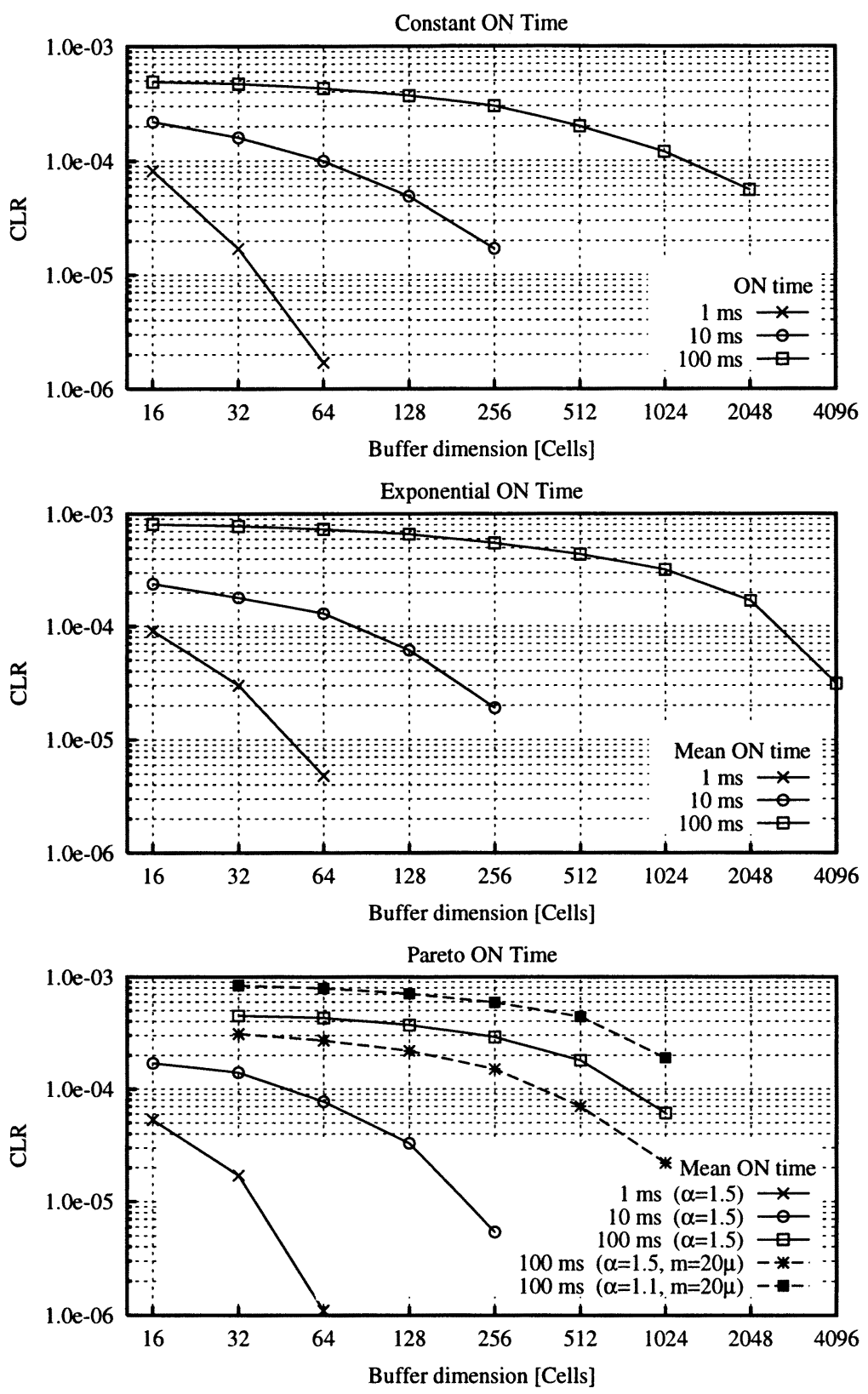

Figure 8 Cell loss ratio versus buffer size for VBR connections when the CAC parameters are set so as to achieve CLR $=10^{-4}$ 
Since the conditions under which CAC algorithms normally operate are rather different from those assumed in Lindberger's analysis, it can be interesting to verify under which conditions the cell loss ratio nominally offered by the CAC is actually achieved.

For this reason we defined the parameters of our CAC algorithms so as to nominally achieve a cell loss ratio equal to $10^{-4}$, obtaining $\alpha=0.926$ and $\gamma_{0}=1.6$, and we simulated the ATM network topology of Fig. 1 at both the call and cell levels.

The simulation at the call level, as usual, provided the configurations (5, as in the provious Section) to be later investigated at the cell level in order to obtain the cell loss ratio results.

The cell-level simulations were run assuming that ON/OFF VBR sources always experience exponentially distributed OFF periods, whereas the distributions of the ON periods were set to be either constant, or exponential, or Pareto, with average equal to either 1 , or 10 , or $100 \mathrm{~ms}$ in the three cases. The parameter $\alpha$ of the Pareto distribution was set to 1.5 .

Results are reported in Fig. 8 as curves of the cell loss ratio versus the buffer size. Simulations have been run for buffer dimension up to 4096 cells: missing points mean that no cell loss was recorded in an overall simulated time of $1.5 \cdot 10^{8}$ slots (roughly $420 \mathrm{~s}$ ).

We can immediately observe that achieving cell loss ratios smaller than $10^{-4}$ is quite difficult, and requires rather large buffers, specially in the cases of long ON periods, that translate in higher correlations in the cell streams. On the other hand the distribution of the ON periods does not seem to have as big an influence, even if non-constant distributions yield a slightly higher CLR.

It could be argued that these results are driven by the fact that our scenario, characterized by a parking lot configuration and by a mix of CBR and VBR sources, is quite different from the bottleneck configuration with only homogeneous VBR sources used for the computation of the equivalent bandwidth in [5]. In order to investigate this possibility we have simulated a single bottleneck, indeed a multiplexer, collecting i.i.d. connections with the same characteristics of the VBR connections used in previous scenarios. Setting the target CLR to $10^{-4}$, Eq. (12) yields a number of admitted connections equal to 537 .

Fig. 9 reports the results obtained in the above scenario when the ON periods are constant, since these are the validity conditions for Eqs. (12)(15). The first comment on these results is the striking similarity between the curves in Fig. 9 and those in the upper plot of Fig. 8. Indeed we observe that in all cases the influence of the ON time average duration is dominant on the CLR: Eqs. (12)-(15) are derived without the explicit knowledge of the ON time duration, so that this behavior can not be caught by this equivalent bandwidth approach. A more detailed exam of the two mentioned plots shows that when connections are multiplexed several times within the network, as in 
the parking lot case, the CLR of connections with long ON times is somewhat reduced, while the CLR of connections with very short ON times $(1 \mathrm{~ms})$ worsens by nearly an order of magnitude: this observation clearly shows that multiplexing is not always beneficial from the CLR point of view.

An additional remark on equivalent bandwidth CAC algorithms can be drawn from Figs. 9 and 8: neither the examined network topologies, nor the ON periods distribution significantly influence the performance of equivalent bandwith CAC algorithms; however the average ON time of connections has a dramatic impact on CLR performance and must be taken into account in CAC algorithms for VBR sources.

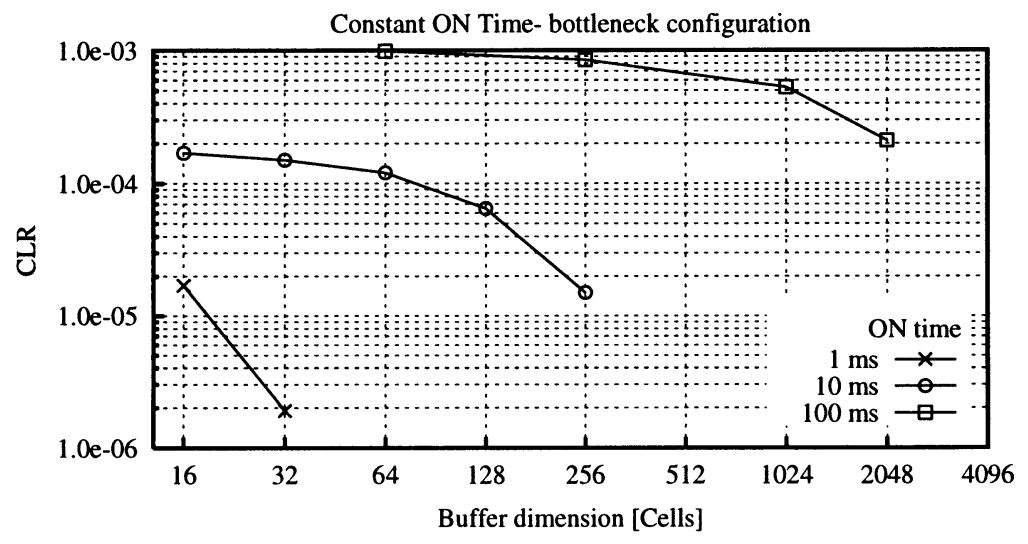

Figure 9 Cell loss ratio versus buffer size for VBR connections in a multiplexer when the CAC parameters are set so as to achieve CLR $=10^{-4}$

The results discussed in this Section indicate that equivalent bandwidth CAC algorithms supporting cell-level performance guarantees should be employed only in those network operating conditions for which they were derived; otherwise, the actual network performance may be remarkably different than expected.

\section{CONCLUSIONS}

We described and evaluated by simulation a simple CAC algorithm for ATM networks supporting different QoS classes.

The CAC algorithm proved to be capable of satisfying the QoS requirements of CBR and VBR connections; moreover, if the ATM network implements the $\mathrm{ABR}$ service class, the CAC algorithm can also guarantee quite a good performance to TCP connections, as well as a very satisfactory exploitation 
of the network resources. A similarly good behavior cannot be achieved with the UBR service class.

The CAC algorithm was shown to be reducible to the equivalent bandwidth CAC algorithm proposed by Lindberger, in the case in which only ON/OFF VBR sources are present in the network. For this latter case some results were obtained showing the scope of validity of the above equivalent bandwidth CAC algorithm.

\section{REFERENCES}

[1] Z.Dziong, B.Shukhman, L.G.Mason, "Estimation of Aggregate Effective Bandwidth for Traffic Admission in ATM Networks", INFOCOM'95, Boston, MA, USA, May 1995

[2] H.M.Vin, A.Goyal, P.Goyal, "An Observation-Based Admission Control Algorithm for Multimedia Servers", ICMCS'94, Boston, MA, USA, April 1994

[3] S.Jamin, P.B.Danzig, S.Shenker and L.Zhang, "A Measurement-based Admission Control Algorithm for Integrated Services Packet Network", SIGCOMM'95, Cambridge, MA, USA, August 1995

[4] J.Roberts, U.Mocci, J.Virtamo (Eds.), "Broadband Network Teletraffic", Final Report of Action COST 242, Lecture Notes in Computer Science - 1155, Springer-Verlag, 1996

[5] K.Lindberger, "Analytical Models for the Traffical Problems with Statistical Multiplexing in ATM Networks", 13th Int. Teletraffic Congress Proceedings, pages 807-813, North Holland - Elsevier Science Publisher, 1991.

[6] ITU-T Reccommendation I.356 "B-ISDN ATM Layer Cell Transfer Performance"

[7] P.Castelli, "Accettazione Delle Connessioni e Supporto di Diverse Classi di Qualità del Servizio", CSELT Working Paper, June 5, 1996 (in Italian).

[8] R.Guerin, H.Ahmadi, M.Naghshineh, "Equivalent Capacity and Its Application to Bandwidth Allocation in High-Speed Networks", IEEE Journal on Selected Areas in Communications, vol.9, pp.968-981, 1991

[9] M.Ajmone Marsan, A.Bianco, C.Casetti, C.F.Chiasserini, A.Francini, R.Lo Cigno, M.Mellia, M.Munafò, "An Integrated Software Environment for the Simulation of ATM Networks", $S C S C^{\prime} 97$, Arlington, VA, USA, July 1997.

[10] R.Jain, S.Kalyanaraman, R.Viswanathan, R.Goyal, "A Sample Switch Algorithm", ATM Forum-TM 95-0178R1, February 1995 


\section{BIOGRAPHIES}

Marco Ajmone Marsan is a Full Professor at the Dipartimento di Elettronica of Politecnico di Torino. He holds a Dr.Ing. degree in Electronic Engineering from Politecnico di Torino, and a Master of Science from the University of California, Los Angeles. He has coauthored over 150 journal and conference papers in the areas of Communications and Computer Science, as well as the two books. His research interests are in the fields of performance evaluation of data communication and computer systems, communication networks and queueing theory. M. Ajmone Marsan is a Senior Member of IEEE.

Andrea Bianco is an Assistant Professor at the Dipartimento di Elettronica of Politecnico di Torino, Italy, since December 1994. He holds a Dr.Ing. degree in Electronics Engineering and a Ph.D. in Telecommunications Engineering both from Politecnico di Torino. His research interests are in the fields of access protocols for all-optical networks and performance analysis of ATM networks.

Claudio Casetti holds a Dr. Ing. degree in Electronics Engineering and a Ph.D. in Electronics and Telecommunications Engineering both from Politecnico di Torino. Since August 1995 until June 1996, he was with the Computer Science Department at the University of Massachusetts at Amherst as visiting researcher. His research interests are in the fields of Call Admission Control in both ATM and IntegratedServices Packet Networks, and of Multicasting in Wormhole Networks.

Paolo Castelli received the degree in electronic engineering from Politecnico di Torino, Italy, in 1985. In 1989 he joined the Network Planning and Management Department in CSELT, where he is now the head of the Traffic Engineering Research Unit. His area of activity includes the analysis of traffic management aspects, with special attention to ATM networks. He has participated in several EURESCOM projects devoted to the specification of ATM networks and he was involved in the standardisation of the B-ISDN in ITU-T SG 13 and ETSI NA5, where he has been "rapporteur" for the traffic control aspects.

Renato Lo Cigno is a Research Engineer at the Dipartimento di Elettronica of Politecnico di Torino. He received a Dr.Ing. degree in Electronics Engeneering from Politecnico di Torino in 1988. Since then he has been with the telecommunication research group of the Dipartimento di Elettronica of Politecnico di Torino. His research interests are in the fields of communication networks simulation and performance analysis.

Marco Mellia holds a Dr. Ing. degree in Electronics Engineering from Politecnico di Torino. Between February and October 1997, he was a Researcher supported by CSELT, developing a Call Admission Control Algoritmh for ATM networks. Since November 1997, he is a PhD student at Dipartimento di Elettronica of Politecnico di Torino. His research interests are in the fields of simulation of High-Speed Networks and Connection Admission Control in ATM Networks.

Maurizio Munafò is a Research Engineer at the Dipartimento di Elettronica of Politecnico di Torino. He received a Dr.Ing. degree in Electronics Engineering since 1991 and a Ph.D. in Telecommunications Engineering, both from Politecnico di Torino. Since November 1994 he is with the Dipartimento di Elettronica of Politecnico di Torino, where he has been involved in the development of an ATM networks simulator. His research interests are in the fields of simulation and performance analysis of communication systems. 
Congestion Control 